\title{
Question 2
}

\section{How can sequence-tagged sites within a DNA sequence be identified?}

\author{
doi:10.1038/ng967
}

The NCBI's electronic PCR (e-PCR) tool ${ }^{12}$, which is part of the UniSTS resource, can be used to find STS markers within a DNA fragment of interest. UniSTS (http://www.ncbi.nih.gov/ genome/sts/) contains all the available data on STS markers, including primer sequences, product size, mapping information and alternative names. Links to other NCBI resources such as Entrez, LocusLink and the MapViewer are also provided. e-PCR looks for potential STSs in a DNA sequence by searching for subsequences with the correct orientation and distance that could represent the PCR primers used to generate known STSs.

The e-PCR home page can be found by going to the NCBI home page, at http://www.ncbi.nlm.nih.gov, and then following the Electronic PCR link in the right-hand column. On the e-PCR home page, paste the sequence of interest or enter an accession number into the large text box at the top of the page. The accession number of the sequence for this example is AF288398. This sequence contains only one STS, stSG47693, which is located between nucleotides (nt) 2102 and 2232 of the sequence under study (Fig. 2.1).

Click on the marker name to bring up details of the STS from UniSTS (Fig. 2.2). The primer information and PCR product size are listed at the top of the page, along with alternative names for the marker. Often STSs are known by different names on differ- ent maps. Cross-references to LocusLink, UniGene and the Genebridge 4 map to which this STS was mapped are shown next. The mapping information section contains links to the NCBI's MapViewer. At the bottom of the page, the Electronic PCR results show other sequences, including contigs, mRNAs and ESTs that may contain this STS marker.

To see the genomic context of the STS marker in all maps to which it has been mapped, click on the link labeled MapViewer at the top of the Mapping Information section. This map view (Fig. 2.3) shows two maps. Note that, in this view, the STS stSG47693 is called RH92759 (highlighted in pink). Gene Map '99-Genebridge 4 (GM99_GB4, left) has 46,000 STS markers mapped onto the GB4 RH panel by the International Radiation Hybrid Consortium. The STS map (right) shows the NCBI's placement of STSs onto the genome sequence assembly using e-PCR. Gray lines connect markers that appear in both maps, whereas the red line denotes where the STS RH92759 appears on both maps. In the region shown, there are a total of 211 STSs on the STS map, but only 20 are labeled in this view. To the right of the STS map, the green and yellow circles show the maps on which the STS markers have been placed. One can zoom in or out of this view by clicking on the lines of the zoom tool in the left sidebar. 


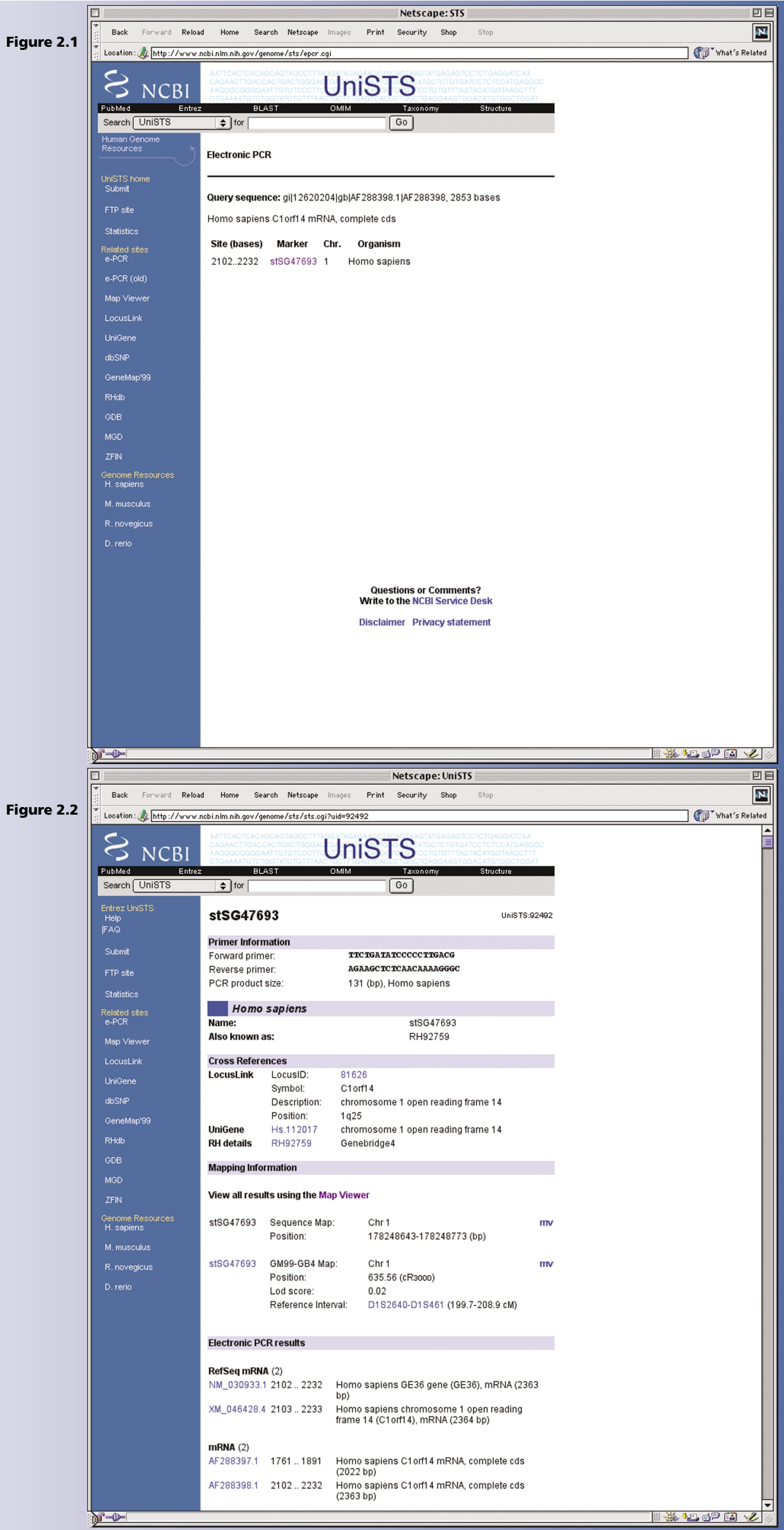


user's guide

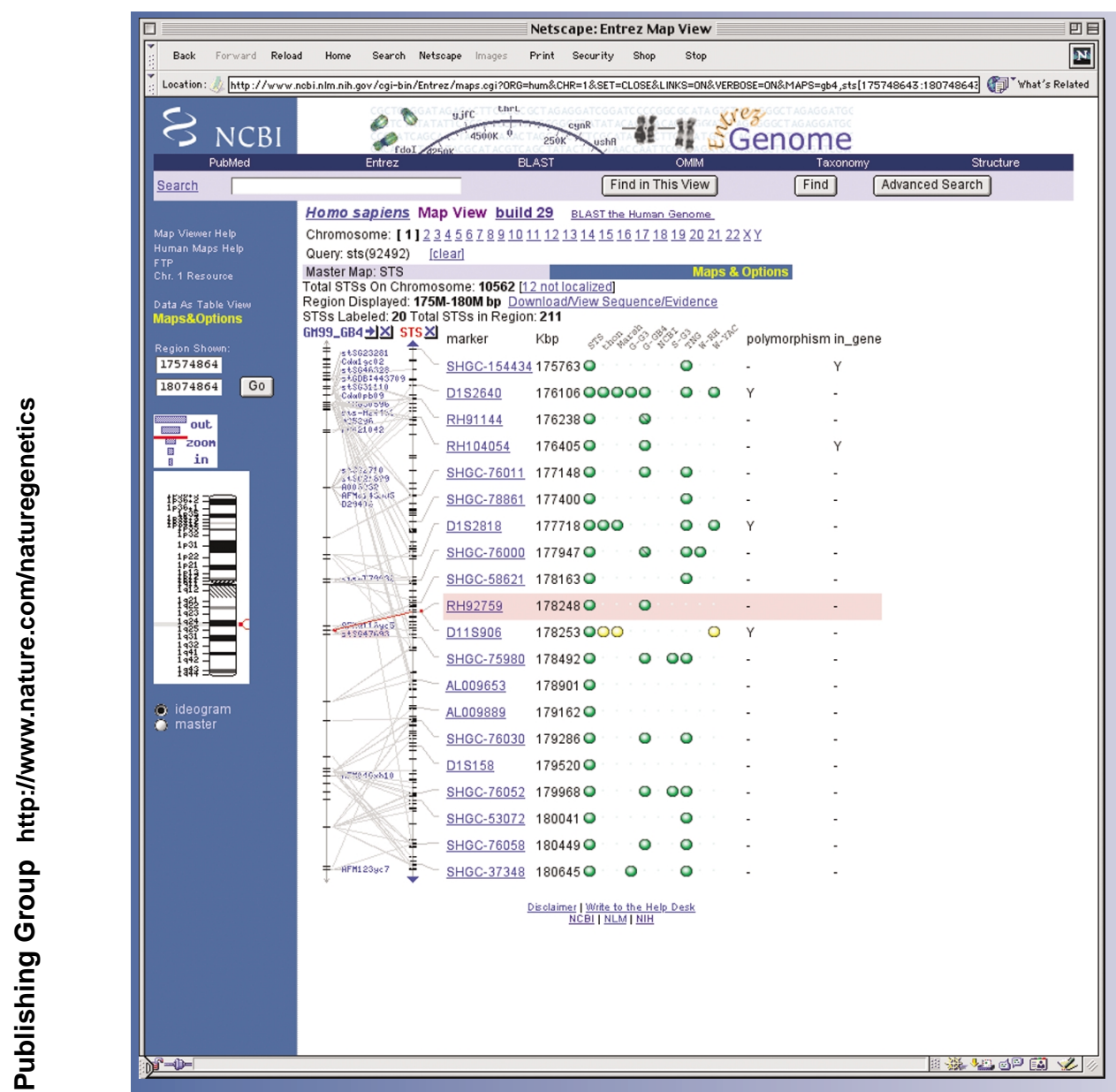

Figure 2.3 